\title{
NONEXISTENCE OF POSITIVE VERY WEAK SOLUTIONS TO AN ELLIPTIC PROBLEM WITH BOUNDARY REACTIONS
}

\section{FUTOSHI TAKAHASHI}

\begin{tabular}{|c|l|}
\hline Citation & OCAMI Preprint Series \\
\hline Issue Date & 2014 \\
\hline Type & Preprint \\
\hline Textversion & Author \\
\hline Relation & $\begin{array}{l}\text { The following article has been submitted to Kodai Mathematical Journal. } \\
\text { This is not the published version. Please cite only the published version. The article } \\
\text { has been published in final form at } \underline{\mathrm{https}: / / \text { doi.org } / 10.2996 / \mathrm{kmj} / 1414674620} .\end{array}$ \\
\hline Is version of & $\underline{\text { https://doi.org/10.2996/kmj/1414674620 } .}$ \\
\hline
\end{tabular}

From: Osaka City University Advanced Mathematical Institute http://www.sci.osaka-cu.ac.jp/OCAMI/publication/preprint/preprint.html 


\title{
NONEXISTENCE OF POSITIVE VERY WEAK SOLUTIONS TO AN ELLIPTIC PROBLEM WITH BOUNDARY REACTIONS
}

\author{
FUTOSHI TAKAHASHI
}

\begin{abstract}
We consider a semilinear elliptic problem with the boundary reaction:

$$
-\Delta u=0 \quad \text { in } \Omega, \quad \frac{\partial u}{\partial \nu}+u=a(x) u^{p}+f(x) \quad \text { on } \partial \Omega,
$$

where $\Omega \subset \mathbb{R}^{N}, N \geq 3$, is a smooth bounded domain with a flat boundary portion, $p>1, a, f \in L^{1}(\partial \Omega)$ are nonnegative functions, not identically equal to zero. We provide a necessary condition and a sufficient condition for the existence of positive very weak solutions of the problem. As a corollary, under some assumption of the potential function $a$, we prove that the problem has no positive solution for any nonnegative external force $f \in L^{\infty}(\partial \Omega), f \not \equiv 0$, even in the very weak sense.
\end{abstract}

\section{INTRODUCTION.}

In this paper, we consider the semilinear elliptic boundary value problem with the boundary reaction:

$$
\begin{cases}-\Delta u=0 & \text { in } \Omega, \\ u \geq 0 & \text { in } \bar{\Omega}, \\ \frac{\partial u}{\partial \nu}+u=a(x) u^{p}+f(x) & \text { on } \partial \Omega\end{cases}
$$

where $\Omega \subset \mathbb{R}^{N}, N \geq 2$ is a smooth bounded domain, $\nu$ is the exterior unit normal vector to $\partial \Omega, p>1, a, f \in L^{1}(\partial \Omega)$ are nonnegative functions, not identically equal to zero.

In the recent paper, Quittner and Reichel [6] consider the more general problem with nonlinear Neumann boundary conditions:

$$
-\Delta u=0 \quad \text { in } \Omega, \quad \frac{\partial u}{\partial \nu}+u=g(x, u) \quad \text { on } \partial \Omega
$$

Date: March 28, 2014.

2010 Mathematics Subject Classification: 35J25. 35J60.

Key words: very weak solution, boundary reaction, nonexistence. 
where $g: \partial \Omega \times \mathbb{R} \rightarrow \mathbb{R}$ is a Carathéodory function. They call a function $u: \bar{\Omega} \rightarrow \overline{\mathbb{R}}$ a very weak solution of the problem (1.2) if $\left.u\right|_{\Omega} \in L^{1}(\Omega)$, $\left.u\right|_{\partial \Omega}, g\left(\cdot,\left.u\right|_{\partial \Omega}\right) \in L^{1}(\partial \Omega)$ and

$$
-\int_{\Omega} u \Delta \zeta d x=\int_{\partial \Omega} g(x, u) \zeta d s_{x}-\int_{\partial \Omega} u\left(\zeta+\frac{\partial \zeta}{\partial \nu} u\right) d s_{x}
$$

holds for any $\zeta \in C^{2}(\bar{\Omega})$. In [6], Quittner and Reichel prove several properties of very weak solutions, such as regularity and the integral representation formula of the very weak solution to (1.2) for the linear case, regularity and a priori bounds in nonlinear case, and so on. See $\S 2$ for some of their results. Moreover, the authors obtain the interesting existence results of singular very weak solutions to the model problem, i.e., $g(x, u)=\left(u_{+}\right)^{p}$ in (1.2), on a special domain $\Omega$ as described below: Let $\Omega \subset \mathbb{R}_{+}^{N}=\left\{x=\left(x^{\prime}, x_{N}\right): x_{N}>0\right\}$ be a smooth bounded domain with a flat boundary portion $\Gamma_{1}$, that is, there exist two closed sets $\Gamma_{1}, \Gamma_{2} \subset \partial \Omega$ such that

(i) $\partial \Omega=\Gamma_{1} \cup \Gamma_{2}, \Gamma_{1} \subset \partial \mathbb{R}_{+}^{N} \cong \mathbb{R}^{N-1}$ and $0 \in \operatorname{int}\left(\Gamma_{1}\right)$,

(ii) $\overline{\operatorname{int}\left(\Gamma_{i}\right)}=\Gamma_{i}$ for $i=1,2$ and $\operatorname{int}\left(\Gamma_{1}\right) \cap \operatorname{int}\left(\Gamma_{2}\right)=\emptyset$.

By perturbing the explicit singular half-space solution and using a variational method, the authors prove the following existence result for the very weak solutions: For $N=3,4$ and $p>\frac{N-1}{N-2}$ but very close to $\frac{N-1}{N-2}$, the problem

$$
-\Delta u=0 \quad \text { in } \Omega, \quad \frac{\partial u}{\partial \nu}+u=u^{p} \quad \text { on } \partial \Omega
$$

admits at least two positive, unbounded very weak solutions, blowing up at $0 \in \operatorname{int}\left(\Gamma_{1}\right)$, see [6]: Theorem 17. Also for any $p>\frac{N-1}{N-2}$, there exists a function $f \in L^{\infty}(\partial \Omega)$ such that the problem

$$
-\Delta u=0 \quad \text { in } \Omega, \quad \frac{\partial u}{\partial \nu}=u^{p}+f(x) \quad \text { on } \partial \Omega
$$

admits a positive, unbounded very weak solution, see [6]: Theorem 12.

However, if we introduce a (singular) potential function into the equation, the situation is drastically changed. Actually, main result in this paper concerns the nonexistence of positive very weak solutions to (1.1) as follows:

Theorem 1.1. Let $\Omega \subset \mathbb{R}^{N}, N \geq 3$, be a smooth bounded domain with $0 \in \partial \Omega$. Let $p>1$ and $f \in L^{\infty}(\partial \Omega), f \geq 0, f \not \equiv 0$ on $\partial \Omega$. Assume that $a \in L^{1}(\partial \Omega)$ be a function such that $a \geq 0$ on $\partial \Omega$, and there exists $R>0$ such that

$$
\int_{B_{R}^{N}(0) \cap \partial \Omega} \frac{a(y)}{|y|^{N-2}} d s_{y}=+\infty
$$


where $B_{R}^{N}(0)$ denotes a $N$-dimensional ball with radius $R$ center 0 . Then there exists no positive solution of the problem

$$
-\Delta u=0 \quad \text { in } \Omega, \quad \frac{\partial u}{\partial \nu}+u=a(x) u^{p}+f(x) \quad \text { on } \partial \Omega,
$$

even in the very weak sense.

By a simple calculation, we check that $a(x)=\frac{1}{|x|}$ satisfies the assumptions in Theorem 1.1 on a smooth bounded domain in $\mathbb{R}^{N}, N \geq 3$, with a flat boundary portion $\Gamma_{1}$. Actually, we compute

$$
\begin{aligned}
& \int_{B_{R}^{N}(0) \cap \partial \Omega} \frac{a(y)}{|y|^{N-2}} d s_{y} \geq \int_{B_{R}^{N}(0) \cap \Gamma_{1}} \frac{d s_{y}}{|y|^{N-1}} \\
& =\int_{B_{R}^{N-1}(0) \cap \Gamma_{1}} \frac{d y^{\prime}}{\left|y^{\prime}\right|^{N-1}}=\left|S^{N-2}\right| \int_{0}^{R} \frac{r^{N-2}}{r^{N-1}} d r=+\infty,
\end{aligned}
$$

where $y=\left(y^{\prime}, 0\right) \in \Gamma_{1}, y^{\prime}=\left(y_{1}, \cdots, y_{N-1}\right)$, and $d y^{\prime}=d y_{1} \cdots d y_{N-1}$. Thus we have the nonexistence of positive very weak solutions to the simple elliptic problem like

$$
-\Delta u=0 \quad \text { in } \Omega, \quad \frac{\partial u}{\partial \nu}+u=\frac{u^{p}}{|x|}+f(x) \quad \text { on } \partial \Omega,
$$

for any nonnegative bounded external force $f \not \equiv 0$ on such a domain when $N \geq 3$. This fact contrasts with the existence results by Quittner and Reichel mentioned above.

This paper is organized as follows: In $\S 2$, we collect several useful facts on very weak solutions, which will be used in later sections. Main source of this part is [6], however, some basic lemmas, such as weak maximum principle or existence of very weak solutions by the method of sub-super solutions, are also proved in this section. In $\S 3$, we provide a necessary condition and a sufficient condition for the existence of very weak solutions to (1.1). The result proved here is an extension of that of Brezis and Cabré [1], to the nonlinear Neumann boundary condition cases. Finally in $\S 4$, we prove Theorem 1.1.

\section{SeVERAl FACts ABOUt VERY WEAK SOlutions.}

In this section, we collect several facts about the very weak solutions which will be useful later. We refer the reader to the paper by Quittner and Reichel [6] for complete descriptions and proofs.

In the following, let $\Omega$ be a smooth bounded domain in $\mathbb{R}^{N}, N \geq 2$ (not necessary with a flat boundary portion). As in [6], we define $L^{1}(\Omega \times \partial \Omega)$ as the space of functions $u: \bar{\Omega} \rightarrow \overline{\mathbb{R}}$ such that $\left.u\right|_{\Omega} \in$ $L^{1}(\Omega)$ and $\left.u\right|_{\partial \Omega} \in L^{1}(\partial \Omega)$. This is a Banach space under the norm 
$\|u\|_{L^{1}(\Omega \times \partial \Omega)}=\|u\|_{L^{1}(\Omega)}+\|u\|_{L^{1}(\partial \Omega)}$, and isomorphic to $L^{1}(\Omega) \times L^{1}(\partial \Omega)$. Note that, generally, $\left.u\right|_{\partial \Omega}$ is not the trace of $\left.u\right|_{\Omega}$. With admitting some ambiguity, we will use the symbol $u$ to denote both $\left.u\right|_{\Omega}$ and $\left.u\right|_{\partial \Omega}$ for simplicity.

First we recall the linear theory developed in [6]. Let $u \in L^{1}(\Omega \times \partial \Omega)$ be a very weak solution to the linear problem

$$
-\Delta u=0 \quad \text { in } \Omega, \quad \frac{\partial u}{\partial \nu}+u=g(x) \quad \text { on } \partial \Omega,
$$

where $g \in L^{1}(\partial \Omega)$. Recall a function $u \in L^{1}(\Omega \times \partial \Omega)$ is a very weak solution of (2.1) if

$$
-\int_{\Omega} u \Delta \zeta d x=\int_{\partial \Omega} g(x) \zeta d s_{x}-\int_{\partial \Omega} u\left(\zeta+\frac{\partial \zeta}{\partial \nu}\right) d s_{x}
$$

holds for any $\zeta \in C^{2}(\bar{\Omega})$.

Next lemma concerns the unique solvability and a priori estimate of very weak solutions to (2.1).

Lemma 2.1. ([6]: Lemma 3) Let $g \in L^{1}(\partial \Omega)$. Then (2.1) admits a unique very weak solution $u \in L^{1}(\Omega \times \partial \Omega)$ with the estimate

$$
\|u\|_{L^{1}(\Omega \times \partial \Omega)} \leq C\|g\|_{L^{1}(\partial \Omega)}
$$

for some $C>0$. Moreover, $u \geq 0$ a.e. (with respect to $N$-dimensional Lebesgue measure) in $\Omega$ and a.e. (with respect to the surface measure on $\partial \Omega$ ) on $\partial \Omega$, if $g \geq 0$ a.e. on $\partial \Omega$.

Next lemma concerns the weak maximum principle.

Lemma 2.2. Let $h, k \in L^{1}(\partial \Omega)$. Let $u \in L^{1}(\Omega \times \partial \Omega)$ denote a very weak supersolution to

$$
-\Delta u \geq 0 \quad \text { in } \Omega, \quad \frac{\partial u}{\partial \nu}+u \geq h \quad \text { on } \partial \Omega,
$$

in the sense that

$$
-\int_{\Omega} u \Delta \zeta d x \geq \int_{\partial \Omega} h \zeta d s_{x}-\int_{\partial \Omega} u\left(\zeta+\frac{\partial \zeta}{\partial \nu}\right) d s_{x}
$$

for any $\zeta \in C^{2}(\bar{\Omega}), \zeta \geq 0$ on $\bar{\Omega}$, and let $v \in L^{1}(\Omega \times \partial \Omega)$ be the unique very weak solution to

$$
-\Delta v=0 \quad \text { in } \Omega, \quad \frac{\partial v}{\partial \nu}+v=k \quad \text { on } \partial \Omega,
$$

respectively. If $h \geq k$ on $\partial \Omega$, then we have $u \geq v$ on $\bar{\Omega}$. 
Proof. For any $\varphi \in C_{0}^{\infty}(\Omega), \varphi \geq 0$, put $\zeta$ be the unique solution to

$$
-\Delta \zeta=\varphi \quad \text { in } \Omega, \quad \frac{\partial \zeta}{\partial \nu}+\zeta=0 \quad \text { on } \partial \Omega .
$$

Then $\zeta \in C^{2}(\bar{\Omega})$ and $\zeta \geq 0$ on $\bar{\Omega}$ by the maximum principle. Testing by $\zeta$ and subtracting, we have

$$
-\int_{\Omega}(u-v) \Delta \zeta d x \geq \int_{\partial \Omega}(h-k) \zeta d s_{x}-\int_{\partial \Omega}(u-v)\left(\zeta+\frac{\partial \zeta}{\partial \nu}\right) d s_{x},
$$

which yields

$$
\int_{\Omega}(u-v) \varphi d x \geq \int_{\partial \Omega}(h-k) \zeta d s_{x} \geq 0 .
$$

Since $\varphi \in C_{0}^{\infty}(\Omega), \varphi \geq 0$ is arbitrary, we have $u-v \geq 0$ a.e. on $\Omega$.

Also, for any $\eta \in C^{\infty}(\partial \Omega), \eta \geq 0$, let $\xi \in C^{2}(\bar{\Omega})$ be the unique solution to

$$
-\Delta \xi=0 \quad \text { in } \Omega, \quad \frac{\partial \xi}{\partial \nu}+\xi=\eta \quad \text { on } \partial \Omega .
$$

Maximum principle implies $\xi \geq 0$ on $\bar{\Omega}$. Testing by $\xi$ and subtracting, we have in this case

$$
\int_{\partial \Omega}(u-v) \eta d s_{x} \geq \int_{\partial \Omega}(h-k) \xi d s_{x} \geq 0 .
$$

Since $\eta \in C^{\infty}(\partial \Omega), \eta \geq 0$ is arbitrary, again we have $u-v \geq 0$ a.e. on $\partial \Omega$.

Lemma 2.3. Let $g: \partial \Omega \times \mathbb{R} \ni(x, s) \mapsto g(x, s) \in \mathbb{R}_{+}$is a nonnegative Carathéodory function, increasing with respect to $s$ for any $x \in \partial \Omega$. Assume (1.2) has a weak supersolution $w \in L^{1}(\Omega \times \partial \Omega)$, in the sense that $g(\cdot, w) \in L^{1}(\partial \Omega)$ and

$$
-\int_{\Omega} w \Delta \zeta d x \geq \int_{\partial \Omega} g(x, w) \zeta d s_{x}-\int_{\partial \Omega} w\left(\zeta+\frac{\partial \zeta}{\partial \nu}\right) d s_{x}
$$

for any $\zeta \in C^{2}(\bar{\Omega}), \zeta \geq 0$ on $\bar{\Omega}$. Then (1.2) has a very weak solution $u \in L^{1}(\Omega \times \partial \Omega)$.

Proof. Proof will be done by a standard monotone iteration argument. See for example, [2]: Lemma 3, or [7]: Lemma 8. Define $w^{(1)}=w \in$ $L^{1}(\Omega \times \partial \Omega)$. By the definition, we have $g\left(\cdot, w^{(1)}\right) \in L^{1}(\partial \Omega)$. Let $w^{(2)}$ be the unique weak solution of

$$
\begin{cases}-\Delta w^{(2)}=0 & \text { in } \Omega, \\ \frac{\partial w^{(2)}}{\partial \nu}+w^{(2)}=g\left(x, w^{(1)}\right) & \text { on } \partial \Omega\end{cases}
$$


obtained by Lemma 2.1. Thus,

$$
\int_{\Omega}(-\Delta \zeta)\left(w^{(1)}-w^{(2)}\right) d x \geq-\int_{\partial \Omega}\left(\frac{\partial \zeta}{\partial \nu}+\zeta\right)\left(w^{(1)}-w^{(2)}\right) d s_{x}
$$

holds for any $\zeta \in C^{2}(\bar{\Omega}), \zeta \geq 0$ on $\bar{\Omega}$. As before, for given $\varphi \in C_{0}^{\infty}(\Omega)$, $\varphi \geq 0$ on $\Omega$, take $\zeta \in C^{2}(\bar{\Omega})$ as the solution of (2.2). Then we have

$$
\int_{\Omega}\left(w^{(1)}-w^{(2)}\right) \varphi d x \geq 0,
$$

and since $\varphi \geq 0$ can be chosen arbitrary, we conclude that $w^{(1)} \geq w^{(2)}$ a.e. on $\Omega$. Similarly, for any $\eta \in C^{\infty}(\partial \Omega), \eta \geq 0$ on $\partial \Omega$, let $\xi \in C^{2}(\bar{\Omega})$, $\xi \geq 0$, be the solution to (2.3). Then we have

$$
0 \geq-\int_{\partial \Omega} \eta\left(w^{(1)}-w^{(2)}\right) d s_{x}
$$

which implies that $w^{(1)} \geq w^{(2)}$ a.e. on $\partial \Omega$. By induction, we obtain

$$
\begin{array}{ll}
w=w^{(1)} \geq w^{(2)} \geq \cdots \geq w^{(n)} \geq \cdots, & \text { a.e. on } \Omega, \\
w=w^{(1)} \geq w^{(2)} \geq \cdots \geq w^{(n)} \geq \cdots, & \text { a.e. on } \partial \Omega .
\end{array}
$$

By Lemma 2.1, we know $w^{(n)} \geq 0$ since $g$ is nonnegative. By the monotone convergence theorem, $w^{(n)}$ converges to $u$ in $L^{1}(\Omega \times \partial \Omega)$. Since $g(x, s)$ is increasing with respect to $s$ for any $x \in \partial \Omega$, we have also $0 \leq g\left(\cdot, w^{(n)}\right) \leq g\left(\cdot, w^{(1)}\right) \in L^{1}(\partial \Omega)$ for any $n \in \mathbb{N}$, which leads to $g(\cdot, u) \in L^{1}(\partial \Omega)$. Finally, it is easy to check that $u$ is a desired weak solution to (1.2).

The following lemma is an integral representation formula of the unique very weak solution to (2.1).

Lemma 2.4. ([6]: Lemma 5) There exists a linear operator $T$ such that $T$ is a bounded, self-map from $L^{p}(\partial \Omega)$ to $L^{p}(\partial \Omega)$ for every $p \in[1,+\infty]$, and if $g \in L^{1}(\partial \Omega)$, then the unique very weak solution $u \in L^{1}(\Omega \times \partial \Omega)$ to (2.1) can be written as

$$
\begin{aligned}
& u(x)=\frac{1}{2 \pi} \int_{\partial \Omega} T g(y) \log |x-y|^{-1} d s_{y}+d_{\Omega} \int_{\partial \Omega} T g(y) d s_{y}, \quad \text { if } N=2, \\
& u(x)=\frac{1}{(N-2)\left|S^{N-1}\right|} \int_{\partial \Omega} T g(y)|x-y|^{2-N} d s_{y}, \quad \text { if } N \geq 3
\end{aligned}
$$

for all $x \in \bar{\Omega}$. Here $d_{\Omega}$ is a constant depending $\Omega$. Furthermore, $T^{-1}$ exists and bounded from $L^{p}(\partial \Omega)$ to $L^{p}(\partial \Omega)$ for every $p \in[1,+\infty]$ when $N \geq 3$. 
The last claim follows from the proof of [6], since $T$ is an inverse operator of a compact perturbation of the identity, and the compact part maps bounded sets of $L^{p}(\partial \Omega)$ to compact sets in $L^{p}(\partial \Omega)$ for every $p \in[1,+\infty]$.

By using the integral representation formula in Lemma 2.4, the following regularity result for very weak solutions to (2.1) is established in $[6]$.

Lemma 2.5. ([6]: Theorem 6) If $g \in L^{p}(\partial \Omega)$ for some $p \in[1, \infty]$, then the followings are true for the very weak solution $u \in L^{1}(\Omega \times \partial \Omega)$ to (2.1).

(i) $q \geq p$ and $\frac{1}{p}-\frac{1}{q}<\frac{1}{N-1} \Longrightarrow u \in L^{q}(\partial \Omega)$,

(ii) $q \geq p$ and $\frac{1}{p}-\frac{N}{(N-1) q}<\frac{1}{N-1} \Longrightarrow u \in L^{q}(\Omega)$,

(iii) $q \geq p$ and $\frac{1}{p}-\frac{N}{(N-1) q}<0 \Longrightarrow u \in W^{1, q}(\Omega)$.

\section{A NECESSARY CONDITION AND A SUfFicient CONDITION FOR THE EXISTENCE OF VERY WEAK SOLUTIONS.}

In this section, we provide a necessary condition and a sufficient condition for the existence of a positive very weak solution to (1.1) on a general bounded smooth domain $\Omega \subset \mathbb{R}^{N}$. The result can be considered as a generalization of that of Brezis-Cabré [1] to the nonlinear Neumann boundary condition case, and Theorem 1.1 is a direct consequence of this. In [1], Brezis and Cabré establishes a necessary condition and a sufficient condition for the existence of positive very weak solutions to the problem

$$
-\Delta u=a(x) u^{p}+f(x) \quad \text { in } \Omega, \quad u=0 \quad \text { on } \partial \Omega,
$$

where $a, f$ are nonnegative $L^{1}(\Omega)$ functions, not identically zero. Our proof is a direct modification of that of Brezis and Cabré to the nonlinear Neumann boundary condition case.

In the following, let $G(h) \in L^{1}(\Omega \times \partial \Omega)$ denote the unique very weak solution to the linear problem

$$
\begin{cases}-\Delta G(h)=0 & \text { in } \Omega, \\ \frac{\partial G(h)}{\partial \nu}+G(h)=h & \text { on } \partial \Omega\end{cases}
$$

for $h \in L^{1}(\partial \Omega)$; see Lemma 2.1.

Theorem 3.1. Let $p>1$. Assume that $f \in L^{1}(\partial \Omega), f \geq 0, f \not \equiv 0$ on $\partial \Omega$, and $a \in L^{1}(\partial \Omega), a \geq 0, a \not \equiv 0$ on $\partial \Omega$. Put $v=G(f) \in L^{1}(\Omega \times \partial \Omega)$. 
(i) If the problem (1.1) has a very weak solution $u \geq 0$, then av $v^{p} \in$ $L^{1}(\partial \Omega)$ and

$$
G\left(a v^{p}\right) \leq\left(\frac{1}{p-1}\right) v \quad \text { in } \bar{\Omega}
$$

holds true.

(ii) If $a v^{p} \in L^{1}(\partial \Omega)$ and

$$
G\left(a v^{p}\right) \leq\left(\frac{p-1}{p}\right)^{p} \frac{1}{p-1} v \text { in } \bar{\Omega}
$$

holds true, then the problem (1.1) has a very weak solution $u \geq 0$. Furthermore, $v \leq u \leq \frac{p}{p-1} v$ holds on $\bar{\Omega}$.

The proof of Theorem 3.1 consists of several lemmas, which are described below.

Lemma 3.2. Let $u, v \in C^{2}(\bar{\Omega}), v>0$ on $\bar{\Omega}$. Let $\phi \in C^{2}(\mathbb{R})$ be a concave function. Then we have

$$
\left(\frac{\partial}{\partial \nu}+1\right)\left\{v \phi\left(\frac{u}{v}\right)\right\}=\phi^{\prime}\left(\frac{u}{v}\right)\left(\frac{\partial u}{\partial \nu}+u\right)+\left\{\phi\left(\frac{u}{v}\right)-\left(\frac{u}{v}\right) \phi^{\prime}\left(\frac{u}{v}\right)\right\}\left(\frac{\partial v}{\partial \nu}+v\right)
$$

on $\partial \Omega$. In particular, if $\frac{\partial v}{\partial \nu}+v \geq 0$ on $\partial \Omega$, then

$$
\left(\frac{\partial}{\partial \nu}+1\right)\left\{v \phi\left(\frac{u}{v}\right)\right\} \geq \phi^{\prime}\left(\frac{u}{v}\right)\left\{\left(\frac{\partial u}{\partial \nu}+u\right)-\left(\frac{\partial v}{\partial \nu}+v\right)\right\}+\phi(1)\left(\frac{\partial v}{\partial \nu}+v\right)
$$

holds.

Proof. By direct computation, we have

$$
\nabla\left\{v \phi\left(\frac{u}{v}\right)\right\}=\phi^{\prime}\left(\frac{u}{v}\right) \nabla u+\left\{\phi\left(\frac{u}{v}\right)-\left(\frac{u}{v}\right) \phi^{\prime}\left(\frac{u}{v}\right)\right\} \nabla v
$$

which implies

$$
\frac{\partial}{\partial \nu}\left\{v \phi\left(\frac{u}{v}\right)\right\}=\phi^{\prime}\left(\frac{u}{v}\right)\left(\frac{\partial u}{\partial \nu}\right)+\left\{\phi\left(\frac{u}{v}\right)-\left(\frac{u}{v}\right) \phi^{\prime}\left(\frac{u}{v}\right)\right\}\left(\frac{\partial v}{\partial \nu}\right)
$$

on $\partial \Omega$. Adding this to the identity

$$
v \phi\left(\frac{u}{v}\right)=\phi^{\prime}\left(\frac{u}{v}\right) u+\left\{\phi\left(\frac{u}{v}\right)-\left(\frac{u}{v}\right) \phi^{\prime}\left(\frac{u}{v}\right)\right\} v
$$

we have (3.3). Since $\phi$ is concave on $\mathbb{R}$, we have $\phi(s)+(1-s) \phi^{\prime}(s) \geq$ $\phi(1)$ for any $s \in \mathbb{R}$. Putting $s=\frac{u}{v}$, we get

$$
\phi\left(\frac{u}{v}\right)-\left(\frac{u}{v}\right) \phi^{\prime}\left(\frac{u}{v}\right) \geq-\phi^{\prime}\left(\frac{u}{v}\right)+\phi(1) .
$$


Thus if $\frac{\partial v}{\partial \nu}+v \geq 0$ on $\partial \Omega$, we obtain (3.4) by inserting the above inequality into (3.3).

Next lemma is a weak form of Lemma 3.2.

Lemma 3.3. Let $\phi \in C^{1}(\mathbb{R})$ be a concave function with $\phi^{\prime}$ is bounded on $\mathbb{R}$. For $h, k \in L^{1}(\partial \Omega), k \geq 0, k \not \equiv 0$ on $\partial \Omega$, let $u, v$ be the unique very weak solution of

$$
-\Delta u=0 \quad \text { in } \Omega, \quad \frac{\partial u}{\partial \nu}+u=h \quad \text { on } \partial \Omega,
$$

and

$$
-\Delta v=0 \quad \text { in } \Omega, \quad \frac{\partial v}{\partial \nu}+v=k \quad \text { on } \partial \Omega,
$$

respectively. Then it holds

$$
\begin{cases}-\Delta\left(v \phi\left(\frac{u}{v}\right)\right) \geq 0 & \text { in } \Omega, \\ \left(\frac{\partial}{\partial \nu}+1\right)\left\{v \phi\left(\frac{u}{v}\right)\right\} \geq \phi^{\prime}\left(\frac{u}{v}\right)(h-k)+\phi(1) k & \text { on } \partial \Omega\end{cases}
$$

in the weak sense. That is, $v \phi\left(\frac{u}{v}\right) \in L^{1}(\Omega \times \partial \Omega), \phi^{\prime}\left(\frac{u}{v}\right)(h-k)+\phi(1) k \in$ $L^{1}(\partial \Omega)$, and

$$
\begin{aligned}
-\int_{\Omega} v \phi\left(\frac{u}{v}\right) \Delta \zeta d x & \geq \int_{\partial \Omega}\left\{\phi^{\prime}\left(\frac{u}{v}\right)(h-k)+\phi(1) k\right\} \zeta d s_{x} \\
& -\int_{\partial \Omega} v \phi\left(\frac{u}{v}\right)\left(\zeta+\frac{\partial \zeta}{\partial \nu}\right) d s_{x}
\end{aligned}
$$

holds for any $\zeta \in C^{2}(\bar{\Omega}), \zeta \geq 0$ on $\bar{\Omega}$.

Proof. First, by mollifying $\phi$, we see that (3.4) holds for $\phi \in C^{1}(\mathbb{R})$ and concave. Following the proof of [1], we approximate $h, k \in L^{1}(\partial \Omega)$ by sequences $h_{n}, k_{n} \in C^{\infty}(\partial \Omega), k_{n} \geq 0, k_{n} \not \equiv 0$. Let $u_{n}$ and $v_{n}$ be the unique classical solutions

$$
-\Delta u_{n}=0 \quad \text { in } \Omega, \quad \frac{\partial u_{n}}{\partial \nu}+u_{n}=h_{n} \quad \text { on } \partial \Omega,
$$

and

$$
-\Delta v_{n}=0 \quad \text { in } \Omega, \quad \frac{\partial v_{n}}{\partial \nu}+v_{n}=k_{n} \quad \text { on } \partial \Omega
$$

respectively. Note that $v_{n}>0$ in $\bar{\Omega}$ by the standard strong maximum principle (see for example, [5] Theorem 2.2). Consider the problem

$$
-\Delta \zeta=1 \quad \text { in } \Omega, \quad \frac{\partial \zeta}{\partial \nu}+\zeta=0 \quad \text { on } \partial \Omega
$$


Multiplying $\zeta$ to the equations satisfied by $u$ and $u_{n}$, and subtracting, we have

$\left|-\int_{\Omega}\left(u_{n}-u\right) \Delta \zeta d x\right|=\left|\int_{\partial \Omega}\left(h_{n}-h\right) \zeta d s_{x}\right| \leq\left\|h_{n}-h\right\|_{L^{1}(\partial \Omega)}\|\zeta\|_{L^{\infty}(\partial \Omega)}$,

which implies $u_{n} \rightarrow u$ in $L^{1}(\Omega)$, and also $v_{n} \rightarrow v$ in $L^{1}(\Omega)$. By using the solution of

$$
-\Delta \eta=0 \quad \text { in } \Omega, \quad \frac{\partial \eta}{\partial \nu}+\eta=1 \quad \text { on } \partial \Omega,
$$

we also obtain that $u_{n} \rightarrow u, v_{n} \rightarrow v$ in $L^{1}(\partial \Omega)$. Since $v_{n}>0$ on $\bar{\Omega}$, $v_{n} \phi\left(u_{n} / v_{n}\right)$ is well defined and $v_{n} \phi\left(\frac{u_{n}}{v_{n}}\right)$ converges to $v \phi\left(\frac{u}{v}\right)$ (up to a subsequence) a.e. on $\Omega$ and $\partial \Omega$. Now, by (5.10) in [1]:

$$
\begin{aligned}
\left|v_{n} \phi\left(\frac{u_{n}}{v_{n}}\right)\right| & =\left|v_{n}\left\{\phi\left(\frac{u_{n}}{v_{n}}\right)-\phi(0)\right\}+\phi(0) v_{n}\right| \\
& =\left|v_{n} \phi^{\prime}\left(\theta \frac{u_{n}}{v_{n}}\right)\left(\frac{u_{n}}{v_{n}}\right)+\phi(0) v_{n}\right| \leq C\left(\left|u_{n}\right|+\left|v_{n}\right|\right),
\end{aligned}
$$

for some $\theta \in(0,1)$, we see that $v_{n} \phi\left(\frac{u_{n}}{v_{n}}\right)$ is dominated by some $L^{1}$ functions in $L^{1}(\Omega)$ and $L^{1}(\partial \Omega)$ respectively, again up to a subsequence. (Recall $\phi^{\prime}$ is bounded). Thus by Dominated Convergence Theorem, we have

$$
v_{n} \phi\left(\frac{u_{n}}{v_{n}}\right) \rightarrow v \phi\left(\frac{u}{v}\right)
$$

in $L^{1}(\Omega)$ and $L^{1}(\partial \Omega)$, respectively. Since it holds that

$$
\begin{aligned}
-\int_{\Omega} v_{n} \phi\left(\frac{u_{n}}{v_{n}}\right) \Delta \zeta d x & \geq \int_{\partial \Omega}\left\{\phi^{\prime}\left(\frac{u_{n}}{v_{n}}\right)\left(h_{n}-k_{n}\right)+\phi(1) k_{n}\right\} \zeta d s_{x} \\
& -\int_{\partial \Omega} v_{n} \phi\left(\frac{u_{n}}{v_{n}}\right)\left(\zeta+\frac{\partial \zeta}{\partial \nu}\right) d s_{x}
\end{aligned}
$$

for any $\zeta \in C^{2}(\bar{\Omega}), \zeta \geq 0$ on $\bar{\Omega}$, passing to the limit in the above and using the boundedness of $\phi^{\prime}$ again, we obtain (3.5).

Now, we prove Theorem 3.1.

Proof. Just as done by Brezis and Cabré [1], we use the concave function

$$
\phi(s)=\int_{1}^{s} \frac{1}{t^{p}} d t=\frac{1}{p-1}\left(1-\frac{1}{s^{p-1}}\right) \quad \text { for } s \geq 1 .
$$

We see $\phi^{\prime}(s) s^{p}=1$ for $s \geq 1, \phi$ is concave, $\phi^{\prime}$ is bounded for $s \geq 1$, $\phi(1)=0, \phi^{\prime}(1)=1$ and $0 \leq \phi(s) \leq \frac{1}{p-1}$. We extend $\phi$ on the interval 
$(-\infty, 1]$ by setting $\phi(s)=s-1$. Then $\phi$ thus obtained satisfies the assumptions in Lemma 3.3.

First, we prove (i) of Theorem 3.1. Assume there exists a very weak solution $u \geq 0$ to (1.1):

$$
-\Delta u=0 \quad \text { in } \Omega, \quad \frac{\partial u}{\partial \nu}+u=a(x) u^{p}+f(x) \quad \text { on } \partial \Omega .
$$

Since $a(x) u^{p}+f(x) \geq f(x)$ on $\partial \Omega$, weak maximum principle (Lemma 2.2) implies

$$
u \geq G(f)=v \quad \text { on } \bar{\Omega} .
$$

Thus $0 \leq a(x) v^{p} \leq a(x) u^{p}$ on $\partial \Omega$ and since $a u^{p} \in L^{1}(\partial \Omega)$ by the definition of the very weak solution $u$, we have $a v^{p} \in L^{1}(\partial \Omega)$.

Then we take $\phi$ as above and apply Lemma 3.3 with $h=a u^{p}+f$, $k=f$. Since $u / v \geq 1$, we obtain

$$
\begin{aligned}
-\int_{\Omega} v \phi\left(\frac{u}{v}\right) \Delta \zeta d x & \geq \int_{\partial \Omega}\left\{\phi^{\prime}\left(\frac{u}{v}\right)\left(a u^{p}+f-f\right)+\phi(1) f\right\} \zeta d s_{x} \\
& -\int_{\partial \Omega} v \phi\left(\frac{u}{v}\right)\left(\zeta+\frac{\partial \zeta}{\partial \nu}\right) d s_{x} \\
& =\int_{\partial \Omega} a v^{p} \zeta d s_{x}-\int_{\partial \Omega} v \phi\left(\frac{u}{v}\right)\left(\zeta+\frac{\partial \zeta}{\partial \nu}\right) d s_{x}
\end{aligned}
$$

for any $\zeta \in C^{2}(\bar{\Omega}), \zeta \geq 0$ on $\bar{\Omega}$ by (3.5). This is the weak form of

$$
\begin{cases}-\Delta\left(v \phi\left(\frac{u}{v}\right)\right) \geq 0 & \text { in } \Omega, \\ \left(\frac{\partial}{\partial \nu}+1\right)\left\{v \phi\left(\frac{u}{v}\right)\right\} \geq a(x) v^{p} & \text { on } \partial \Omega,\end{cases}
$$

therefore, again by the weak maximum principle Lemma 2.2, we have

$$
G\left(a v^{p}\right) \leq v \phi\left(\frac{u}{v}\right) \leq \frac{1}{p-1} v
$$

here we have used $\phi(s) \leq \frac{1}{p-1}$ for $s \geq 1$. This proves (3.1) of part (i).

Next, we prove (ii) of Theorem 3.1. Assume $a v^{p} \in L^{1}(\partial \Omega)$ and (3.2) holds. Put

$$
w=\left(\frac{p}{p-1}\right)^{p} G\left(a v^{p}\right)+v \in L^{1}(\Omega \times \partial \Omega) .
$$

Then $-\Delta w=0$ and (3.2) implies that $w \leq \frac{p}{p-1} v$, thus

$$
\left(\frac{\partial}{\partial \nu}+1\right) w=\left(\frac{p}{p-1}\right)^{p} a v^{p}+f \geq a w^{p}+f
$$

on $\partial \Omega$. That is, $w$ is a very weak supersolution to (1.1). Always 0 is a subsolution to (1.1). Therefore by the monotone iteration Lemma 2.3, 
we have a very weak solution $u$ satisfying $0 \leq u \leq w$. Actually, weak maximum principle implies

$$
v \leq u \leq w \leq \frac{p}{p-1} v
$$

This proves part (ii) of Theorem.

Remark 3.4. Similar results as Theorem 3.1 hold true for other variants of the problem (1.1), which are considered in [4], [3], or [7], [8]. For example, the following claim can be proved in the same way as before.

Let $p>1$ and a, $f$ satisfy the same assumption of Theorem 3.1. Consider the problem

$$
-\Delta u+u=0 \quad \text { in } \Omega, \quad \frac{\partial u}{\partial \nu}=a(x) u^{p}+f(x) \quad \text { on } \partial \Omega
$$

on a smooth bounded domain $\Omega$. The notion of very weak solutions and the corresponding linear theory (existence, uniqueness, and so on) are examined in [7]. For any $h \in L^{1}(\partial \Omega)$, let $G(h) \in L^{1}(\Omega \times \partial \Omega)$ be the unique very weak solution of

$$
-\Delta G(h)+G(h)=0 \quad \text { in } \Omega, \quad \frac{\partial G(h)}{\partial \nu}=h \quad \text { on } \partial \Omega .
$$

We have:

(i) If the problem (3.6) has a very weak solution $u \geq 0$, then $a G(f)^{p} \in$ $L^{1}(\partial \Omega)$ and

(ii) If $a G(f)^{p} \in L^{1}(\partial \Omega)$ and

$$
G\left(a G(f)^{p}\right) \leq\left(\frac{1}{p-1}\right) G(f) \quad \text { in } \bar{\Omega}
$$

$$
G\left(a G(f)^{p}\right) \leq\left(\frac{p-1}{p}\right)^{p} \frac{1}{p-1} G(f) \text { in } \bar{\Omega},
$$

then the problem (3.6) has a very weak solution $u \geq 0$ with $G(f) \leq$ $u \leq \frac{p}{p-1} G(f)$ on $\bar{\Omega}$.

\section{Proof of Theorem 1.1.}

In this section, we prove Theorem 1.1.

Proof. Assume the contrary that there exists a very weak solution $u \geq$ 0 to $(1.1)$ on a bounded smooth domain $\Omega$ with $0 \in \partial \Omega$. Consider $v=G(f)$, i.e., the unique weak solution of

$$
-\Delta v=0 \quad \text { in } \Omega, \quad \frac{\partial v}{\partial \nu}+v=f \quad \text { on } \partial \Omega .
$$


We know that $v \geq 0, v \not \equiv 0$ on $\bar{\Omega}$ since $f \geq 0, f \not \equiv 0$ on $\partial \Omega$. Note that, by the definition of very weak solutions, $v$ is a distributional solution of

$$
-\Delta v=0 \quad \text { in } \mathcal{D}^{\prime}(\Omega)
$$

(Simply we restrict the class of test functions from $C^{2}(\bar{\Omega})$ to $\mathcal{D}(\Omega)$ ). Thus by Weyl's lemma, we see $v \in C^{\infty}(\Omega)$. Also since $f \in L^{\infty}(\Omega)$, the regularity Theorem 2.5 implies that $v \in W^{1, q}(\Omega)$ for any $q<\infty$, thus $v \in C^{0, \alpha}(\bar{\Omega})$ for some $\alpha \in(0,1)$. Hence by the standard strong maximum principle (see for example, [5]), we have $v>0$ on $\bar{\Omega}$. We have checked that there exists $c>0$ such that $v \geq c>0$ on $\partial \Omega$, since $v \in C(\partial \Omega)$ and $\partial \Omega$ is compact. Now, let us consider $\tilde{w}=G\left(a c^{p}\right)$, i.e., the unique weak solution of

$$
-\Delta \tilde{w}=0 \quad \text { in } \Omega, \quad \frac{\partial \tilde{w}}{\partial \nu}+\tilde{w}=c^{p} a(x) \quad \text { on } \partial \Omega .
$$

Since $a(x) v^{p} \geq a(x) c^{p}$ on $\partial \Omega, w=G\left(a v^{p}\right)$ satisfies $w \geq \tilde{w}$ by the weak maximum principle Lemma 2.2. By the integral representation formula Lemma 2.4, $\tilde{w}$ can be written as

$$
\tilde{w}(x)=\frac{c^{p}}{(N-2)\left|S^{N-1}\right|} \int_{\partial \Omega} T a(y)|x-y|^{2-N} d s_{y},
$$

where $T: L^{q}(\partial \Omega) \rightarrow L^{q}(\partial \Omega)$ is a bounded linear operator with a bounded inverse for any $q \in[1,+\infty]$. In the following, we prove that $\tilde{w}(x) \rightarrow+\infty$ as $|x| \rightarrow 0$. Since $T$ and $T^{-1}$ are bounded linear from $L^{q}(\partial \Omega)$ to $L^{q}(\partial \Omega)$ for any $q \in[1,+\infty]$, it is enough to prove that

$$
\int_{\partial \Omega} \frac{a(y)}{|x-y|^{N-2}} d s_{y} \rightarrow+\infty \quad \text { as }|x| \rightarrow 0 .
$$

This is assured by the assumption (1.4), because for $|x| \leq \frac{1}{n}$, we have

$$
\begin{aligned}
\int_{\partial \Omega} \frac{a(y)}{|x-y|^{N-2}} d s_{y} & \geq \int_{B_{R}^{N}(0) \cap \partial \Omega} \frac{a(y)}{|x-y|^{N-2}} d s_{y} \\
& \geq \int_{B_{R}^{N}(0) \cap \partial \Omega} \frac{a(y)}{\left(|y|+\frac{1}{n}\right)^{N-2}} d s_{y} \rightarrow+\infty
\end{aligned}
$$

as $n \rightarrow \infty$. Thus we obtain $w=G\left(a v^{p}\right)$ also blows up as $|x| \rightarrow 0$. However, this contradicts to the necessary condition (3.1) proved in Theorem 3.1:

$$
w=G\left(a v^{p}\right) \leq \frac{1}{p-1} v \quad \text { on } \bar{\Omega},
$$

since $v \in C(\bar{\Omega})$. This proves Theorem 1.1. 


\section{Acknowledgement.}

Part of this work was supported by JSPS Grant-in-Aid for Scientific Research (B), No. 23340038, and JSPS Grant-in-Aid for Challenging Exploratory Research, No. 24654043.

\section{REFERENCES}

[1] H. Brezis and X. Cabré: Some simple nonlinear PDE's without solutions, Boll. Unione Mat. Ital. Sez. B Artic. Ric. Mat. (8) 1, 223-262, (1998)

[2] H. Brezis, T. Cazenave, Y. Martel and A. Ramiandrisoa: Blow up for $u_{t}-$ $\Delta u=g(u)$ revisited, Adv. Differential Equations. 1, 73-90, (1996)

[3] J. Dávila: Singular solutions of semi-linear elliptic problems, Handbook of differential equations: stationary partial differential equations. Vol. VI, 83$176,(2008)$

[4] J. Dávila, L. Dupaigne, and M. Montenegro: The extremal solution of a boundary reaction problem, Commun. Pure Appl. Anal. 7, 795-817, (2008)

[5] J. López-Gḿez: The strong maximum principle, RIMS Kôkyûroku Bessatsu. B15, 113-123, (2009)

[6] P. Quittner, and W. Reichel: Very weak solutions to elliptic equations with nonlinear Neumann boundary conditions, Calc. Var. 32, 429-452, (2008)

[7] F. Takahashi: Extremal solutions to Liouville-Gelfand type elliptic problems with nonlinear Neumann boundary conditions, to appear in Commun. Contemp. Math., preprint available at http://www.sci.osakacu.ac.jp/math/OCAMI/preprint/index.html

[8] F. Takahashi: Continuum spectrum for the linearized extremal eigenvalue problems with boundary reactions, to appear in Proceedings of Equadiff 13, Special issues of Math. Bohemica, preprint available at http://www.sci.osakacu.ac.jp/math/OCAMI/preprint/index.html

\section{Authors' addresses:}

Futoshi Takahashi,

Department of Mathematics, Osaka City University, Osaka, Japan.

e-mail: futoshi@sci.osaka-cu.ac.jp 